\title{
SIMPLIFIED $L^{\infty}$ ESTIMATES FOR DIFFERENCE SCHEMES FOR PARTIAL DIFFERENTIAL EQUATIONS
}

\author{
WILLIAM J. LAYTON
}

\begin{abstract}
L^{\infty}$ estimates for one-step difference approximations to the Cauchy problem for $\partial u / \partial t=\partial u / \partial x$ are proven by means of simple $L^{2}$-techniques. It is shown that, provided the difference approximation is stable in $L^{2}$ (and not necessarily $L^{\infty}$ ) and accurate of order $r$, the error in approximating smooth solutions is $O\left(h^{r}\right)$. This has been proven by Hedstrom and Thomée using Fourier multipliers and Besov spaces. The present paper shows how convergence rates in $L^{\infty}$ can be recovered using simple techniques (such as the Fourier inversion formula). The methods of Hedstrom and Thomée give sharper results when the difference scheme diverges. The present paper exploits the fact that estimates between $L^{\infty}$ and $L^{1}$ are frequently easier to obtain than between $L^{\infty}$ and $L^{\infty}$.
\end{abstract}

1. Introduction. In this paper the problem of approximating solutions to the initial value problem

$$
\frac{\partial u}{\partial t}=\frac{\partial u}{\partial x}, \quad u(x, 0)=u_{0}(x), \quad-\infty<x<\infty
$$

by the 1-step difference scheme

$$
\begin{gathered}
v_{n+1}(x)=A v_{n}(x), \quad v_{0}(x)=u_{0}(x), \\
A v_{n}(x)=\sum_{j \in \mathbf{Z}} a_{j} v(x+j h), \quad \sum_{j \in \mathbf{Z}}\left|a_{j}\right|<\infty,
\end{gathered}
$$

is considered. Here $h, k>0, \lambda=k / h$ is held fixed and $v_{n}(x) \approx u(x, n k)$. A new and simple method of proving that the pointwise error in the approximation (2) is of optimal order is introduced. Previous methods using multiplier theory and Besov spaces involve long calculations and delicate estimates. Those methods based upon Sobolev's Theorem result in a suboptimal estimate for the error unless additional smoothness is assumed. The new method uses simple $L^{2}$ techniques, proves optimal orders of convergence of $v_{n}$ to $u$ under sharp smoothness conditions, and applies to difference operators that are unstable in $L^{\infty}$. In contrast to the estimates arising from multiplier theory, the smoothness conditions upon $u_{0}$ in the new method are stated in $L^{1}$.

The difference scheme (2) is said to be stable in $L^{p}(1 \leqslant p \leqslant \infty)$ if

$$
\left\|A^{n}\right\|_{p} \leqslant C<\infty, \quad n=1,2, \ldots,
$$

Received by the editors August 31, 1981 and, in revised form, February 15, 1982. 1980 Mathematics Subject Classification. Primary 65M15; Secondary 65M10. 
where $C$ is a constant independent of $n$ and $\left\|A^{n}\right\|_{p}$ is the operator norm on $L^{p}(\mathbf{R})$. (2) is said to be accurate of order $r$ if, for every sufficiently smooth solution, $u(x, t)$, to $(1)$,

$$
u(x,(n+1) k)=A u(x, n k)+O\left(h^{r+1}\right) .
$$

If (2) is accurate of order $r>0$, then (2) is consistent with (1).

Part of the Lax equivalence theorem states that if (2) is stable in $L^{p}$ and consistent with (1) then $v_{n} \rightarrow u$ in $L^{p}$ as $h, k \rightarrow 0$. Further, it is well known that $A$ is stable in $L^{2}$ if and only if its symbol $a(\theta)=\sum_{j \in \mathbf{Z}} a_{j} e^{i j \theta}$ satisfies $|a(\theta)| \leqslant 1$ for $|\theta| \leqslant \pi$.

The question of stability and convergence of a difference scheme in $L^{p}$ has recently been studied by a number of people (e.g. Hedstrom [3], Serdyukova [5], Setter [6], Strang [7], Thomée [8], and the book [2] by Brenner, Thomée and Wahlbin). In Hedstrom [3] and Brenner, Thomée and Wahlbin [2] it is shown that if $A$ is stable in $L^{2}$, accurate of order $r$ and if $u$ is sufficiently smooth (measured in an appropriate Besov space), then $v_{n} \rightarrow u$ in $L^{\infty}$. Convergence is optimal with respect to the rate of convergence and the smoothness required of $u_{0}$.

These estimates require extensive use of Fourier multipliers (in particular, the Carlson-Beurling inequality) and Besov spaces. In this paper convergence estimates in the spirit of [ 2 and 3] are shown under different hypotheses than [ 2 and 3] using elementary properties of the Fourier transform and $L^{2}$. The techniques of $[2,3$ and 8] will provide information not furnished here, i.e., the rate of growth of $\left\|A^{n}\right\|_{\infty}$ in the unstable case. Specifically, in Brenner, Thomée and Wahlbin [2] and Hedstrom [3] it is shown that if $|a(\theta)| \equiv 1$ and $\log a(\theta)$ is an analytic function that is not linear then,

$$
\left\|v_{n}\right\|_{L^{\infty}} \geqslant c n^{1 / 2}\left\|u_{0}\right\|, \quad c=\text { constant }>0,
$$

holds for some $u_{0} \in L^{\infty}$.

If $\hat{u}_{0}$ denotes the Fourier transform of $u_{0}$, then $v_{n}(x)$ may be written

$$
v_{n}(x)=(2 \pi)^{-1 / 2} \int_{\mathbf{R}} a(h \theta)^{n} \hat{u}_{0}(\theta) e^{i x \theta} d \theta .
$$

In contrast to the above instability result, for $\hat{u}_{0} \in L^{1}$ and $|a(\theta)| \leqslant 1,\left|v_{n}(x)\right| \leqslant$ $(2 \pi)^{-1 / 2}\left\|\hat{u}_{0}\right\|_{L^{1}}$ follows easily. Thus, if (2) is stable in $L^{2}$ it will be stable in $L^{\infty}$ for this set of initial data.

This idea of obtaining estimates between $L^{\infty}$ and $L^{1}$ is used to find the rate of convergence of $v_{n}$ to $u$ when $u_{0}$ is smooth. The smoothness requirements in $L^{1}$ are sharp in that $\left\|v_{n}-u\right\|_{L^{\infty}}=O\left(h^{r}\right)$, provided $\hat{u}_{0}(\theta) \rightarrow 0$ at $\pm \infty$ in $L^{1}$ like $\theta^{-(r+1)}$.

The following theorem is proven in this paper.

THEOREM. Assume that the difference scheme (2) is stable in $L^{2}$ and accurate of order $r$. Then, for $t=n k>0$,

$$
\left\|u(\cdot, t)-v_{n}\right\|_{L^{\infty}} \leqslant C t h^{r}\left\|u_{0}\right\|_{r+1,1}
$$

where $C$ is a constant independent of $t$ and $h$.

Here the norm $\|\cdot\|_{r+1,1}$, introduced in $\S 2$, is a measure of smoothness of $u_{0}$ in the transform space. It is important that the theorem applies to difference schemes 
stable in $L^{2}$ (and not necessarily $L^{\infty}$ ) since a difference scheme for (1) is stable in $L^{\infty}$ only under very restrictive conditions (see Hedstrom [3], Thomée [8]).

2. Preliminaries. For $1 \leqslant P \leqslant \infty, L^{P}=L^{P}(\mathbf{R})$ denotes the $L^{P}$ space of Lebesgue measurable functions with $L^{P}$ norm, \|\|$_{P}$, defined in the usual manner (see Nikol'skii [4]), finite. Define the Fourier and inverse Fourier transform of a function $f(x), g(\theta)$ as

$$
\begin{gathered}
(F f)(\theta)=\hat{f}(\theta)=(2 \pi)^{-1 / 2} \int_{\mathbf{R}} f(x) e^{-i x \theta} d x, \\
\left(F^{-1} g\right)(x)=\check{g}(x)=(2 \pi)^{-1 / 2} \int_{\mathbf{R}} g(\theta) e^{i x \theta} d \theta .
\end{gathered}
$$

Then $\hat{u}$ and $\hat{v}_{n}$, where $u$ and $v_{n}$ are given by (1) and (2) respectively, satisfy

$$
\hat{u}(\theta, t)=\exp (t i \theta) \hat{u}_{0}(\theta), \quad \hat{v}_{n}(\theta)=a(h \theta)^{n} \hat{u}_{0}(\theta) \text {. }
$$

The inequality

$$
|e(x)| \leqslant(2 \pi)^{-1 / 2}\|\hat{e}\|_{1}
$$

follows easily from the Fourier inversion formula and will play a key role in the analysis that follows. The smoothness conditions will be stated in terms of the $L^{s, p}$ spaces. Define, e.g. following Chapter 9 of Nikol'skii [4], the Bessel-MacDonald potential operator $I_{s}$,

$$
I_{s} u=F^{-1}\left(\left(1+|\theta|^{2}\right)^{-s / 2}(F u)(\theta)\right), \quad-\infty<s<\infty .
$$

$I_{s} u$ can be written as an integral operator, specifically, as convolution of $u$ with the Bessel-MacDonald kernel. Define the $L^{s, p}$ norm as

$$
\|u\|_{s, p}=\left\|F\left(I_{-s} u\right)\right\|_{p}, \quad-\infty<s<\infty .
$$

$L^{s, p}$ is related to the Liouville classes $L_{p}^{s}$ whose norm is given by $\left\|I_{-s} u\right\|_{p}$ (in particular, for $p=2$ the two coincide), see Chapter 9 of Nikol'skii [4] for the latter.

3. Proof of the Theorem. The difference scheme (2) is assumed stable and accurate of order $r$. These two conditions are equivalent to:

(i) $|a(\theta)| \leqslant 1$, for $|\theta| \leqslant \pi$,

(ii) $a(\theta)=\exp (i \lambda \theta+\psi(\theta))$, where $\psi(\theta)=O\left(\theta^{r+1}\right)$ as $\theta \rightarrow 0$, respectively.

(i) is the well-known von Neumann condition and (ii) can be found in Thomé [8].

For $t=n k$, let $e_{n}(x)=u(x, t)-v_{n}(x)$. Then, by virtue of (3),

$$
\hat{e}_{n}(\theta)=\left(\exp (t i \theta)-a(h \theta)^{n}\right) \hat{u}_{0}(\theta) .
$$

The pointwise estimate will follow from the inequality (4),

$$
\begin{aligned}
& (2 \pi)^{1 / 2}\left|e_{n}(x)\right| \leqslant\left\|\hat{e}_{n}\right\|_{1}=\int_{\mathbf{R}}\left|\exp (t i \theta)-a(h \theta)^{n}\right|\left|\hat{u}_{0}(\theta)\right| d \theta \\
& \quad \leqslant\left(\int_{[-\pi / h, \pi / h]}+\int_{\mathbf{R} \backslash-\pi / h, \pi / h]}\right)\left|\exp (t i \theta)-a(h \theta)^{n} \| \hat{u}_{0}(\theta)\right| d \theta .
\end{aligned}
$$


The first integral will be estimated using accuracy (i.e. condition (ii)) and the second will converge to zero rapidly as $h \rightarrow 0$ because of stability and smoothness of $u_{0}$.

Consider now,

$$
\begin{gathered}
\int_{\mathbf{R} \backslash-\pi / h, \pi / h]}\left|\exp (t i \theta)-a(h \theta)^{n}\right|\left|\hat{u}_{0}(\theta)\right| d \theta \\
\leqslant 2 \int_{\mathbf{R} \bigvee-\pi / h, \pi / h]}\left|\hat{u}_{0}(\theta)\right| d \theta
\end{gathered}
$$

For $|\theta| \geqslant \pi h^{-1}$ and $s \geqslant 0,\left(\pi h^{-1}\right)^{-s}(1+|\theta|)^{s / 2} \geqslant 1$. Thus,

$$
\int_{\mathbf{R} \bigvee-\pi / h, \pi / h]}\left|\hat{u}_{0}(\theta)\right| d \theta \leqslant h^{s} \pi^{-s} \int_{\mathbf{R}\lceil-\pi / h, \pi / h]}\left(1+|\theta|^{2}\right)^{s / 2}\left|\hat{u}_{0}(\theta)\right| d \theta,
$$

and the estimate

$$
\int_{\mathbf{R} \bigvee-\pi / h, \pi / h]}\left|\exp (t i \theta)-a(h \theta)^{n}\left\|\hat{u}_{0}(\theta) \mid d \theta \leqslant C h^{s}\right\| u_{0} \|_{s, 1}\right.
$$

follows for any $s, 0 \leqslant s<\infty$. Here, $C=2 \pi^{-s}$ is sufficient.

To estimate the integral over $[-\pi / h, \pi / h]$ note that for $t=n k>0$

$$
a(h \theta)^{n}-\exp (t i \theta)=n \psi(h \theta) \int_{0}^{1} \exp (s(i \theta t+n \psi(h \theta))+(1-s) t i \theta) d s .
$$

Since $|a(\theta)| \leqslant 1, \operatorname{Re}(\psi(h \theta)) \leqslant 0$. Thus,

$$
\left|\exp (t i \theta)-a(h \theta)^{n}\right| \leqslant n|\psi(h \theta)| \leqslant C n h^{s+1}|\theta|^{s+1}
$$

holds for $0 \leqslant s \leqslant r$. Here property (ii) has been used. Applying this estimate to the integral under consideration yields

$$
\begin{aligned}
\int_{[-\pi / h, \pi / h]} \mid & \exp (t i \theta)-a(h \theta)^{n}|| \hat{u}_{0}(\theta) \mid d \theta \\
& \leqslant C n h^{s+1} \int_{[-\pi / h, \pi / h]}|\theta|^{r+1}\left|\hat{u}_{0}(\theta)\right| d \theta \\
& \leqslant C n h^{s+1}\left\|u_{0}\right\|_{s+1,1}, \quad 0 \leqslant s \leqslant r .
\end{aligned}
$$

(5), (7) and (9) together imply that for any $s, 0 \leqslant s \leqslant r$,

$$
\left|e_{n}(x)\right| \leqslant C n h^{s+1}\left\|u_{0}\right\|_{s+1,1}+C h^{s}\left\|u_{0}\right\|_{s, 1} .
$$

Since $n k=t$ and $k / h=\lambda=$ constant, the theorem follows.

4. Remarks. The same idea can be used to derive $L^{\infty}$ estimates of optimal order for any one step, constant coefficient difference scheme approximating the solution to the Cauchy problem for a well-posed constant coefficient partial differential equation in $\mathbf{R}^{n}$. The extension is almost immediate. A close look at the proof reveals that if $u_{0} \in L^{s+1,1}$ for any $s, 0 \leqslant s \leqslant r$, then the error in $L^{\infty}$ will be correspondingly larger- $O\left(h^{s}\right)$, instead of $O\left(h^{r}\right)$. 


\section{REFERENCES}

1. R. A. Adams, Sobolev spaces, Academic Press, New York, 1975.

2. P. Brenner, V. Thomée and L. Wahlbin, Besov spaces and applications to difference methods for initial value problems, Springer-Verlag, New York, 1975.

3. G. W. Hedstrom, The rate of convergence of some difference schemes, SIAM J. Numer. Anal. 5 (1968), $363-406$.

4. S. M. Nikol'skii, Approximation of functions of several variables and embedding theorems, SpringerVerlag, New York, 1975.

5. S. I. Serdyukova, A study of stability in $C$ of explicit difference schemes with constant real coefficients which are stable in $l_{2}$, Ž. Vyčisl. Mat. i Mat. Fiz. 3 (1963), 365-370.

6. H. J. Setter, Maximum bounds for the solutions of initial value problems for partial difference equations, Numer. Math. 5 (1963), 388-424.

7. W. G. Strang, Polynomial approximation of Bernstein type, Trans. Amer. Math. Soc. 105 (1962), 525-535.

8. V. Thomée, Stability of difference schemes in the maximum-norm, J. Differential Equations 1 (1965), $273-292$.

School of Mathematics, Georgia Institute of Technology, Atlanta, Georgia 30332

Current address: Mathematisch Instituut, Katholieke Universiteit, Nijmegen, The Netherlands 